\title{
Silver nanoparticles of variable morphology synthesized in aqueous foams as novel templates
}

\author{
SAIKAT MANDAL, SUJATHA K ARUMUGAM, RENU PASRICHA and \\ MURALI SASTRY* \\ Materials Chemistry Division, National Chemical Laboratory, Pune 411 008, India
}

MS received 25 February 2005

\begin{abstract}
In this paper, we describe the synthesis of silver nanocrystals within aqueous foams as a template. More specifically, we show that aqueous $\mathrm{Ag}^{+}$ions may be electrostatically complexed with the anionic surfactants aerosol OT (sodium bis-2-ethylhexyl-sulfosuccinate, (AOT) and sodium dodecyl sulphate (SDS)) in a highly stable liquid foam. After drainage of the foam, the silver ions are reduced in situ by introducing sodium borohydride into the foam by capillary flow. This leads to the formation of silver nanoparticles of spherical, tape- and sheet-like morphology in the foam. The structure of the foam is extremely complex and presents reaction sites of different spatial extent. The differences in foam reaction-site geometry are believed to be responsible for the morphology variation in the silver nanoparticles observed. The silver nanoparticles are observed to be extremely stable in solution suggesting that the AOT or SDS molecules stabilize them. This approach appears promising for application in large-scale synthesis of nanoparticles and may be readily extended to other chemical compositions.
\end{abstract}

Keywords. Composites; chemical synthesis; intercalation; foam; nanoparticles.

\section{Introduction}

The size and shape-dependent properties of nanomaterials provide a challenge to synthetic chemists for obtaining highly functional advanced materials. It is well known that the shape and size of inorganic nanocrystals control their widely varying electrical, optical and catalytic properties (Alivisatos 1996; Huang and Murray 2001; Kamat 2002). El-Sayed (2001) has shown that cubic platinum nanoparticles with their surfaces bound by $\{100\}$ facets prefer to adsorb hydrogen molecules, while carbon monoxide tends to interact strongly with buckyball-shaped platinum nanoparticles bound by $\{210\}$ facets. Consequently, one of the emerging challenges in materials synthesis is achieving control over the morphology of nanocrystals. One can achieve shape control by the use of a static template to enhance the growth rate of one crystallographic face over another. For example, two-dimensional films of GaAs are obtained when there is favourable epitaxy on a substrate (Cho 1999) as is the case of calcite growth on carboxyl-terminated lipid bilayers (Berman 1995). Hu and co-workers have synthesized one-dimensional Si nanowires by the vapour-liquid-solid growth mechanism using a catalyst (Hu et al 1999). There are also many demonstrated cases of anisotropic inorganic nanocrystal growth in liquid media. Swami and co-workers have recently

\footnotetext{
*Author for correspondence (m.sastry@ncl.res.in)
}

demonstrated the spontaneous reduction of chloroaurate ions present in the aqueous subphase by Langmuir monolayers of hexadecylamine molecules that resulted in the formation of highly oriented, flat gold nanosheets/ nanoribbons bound to the monolayer (Swami et al 2003). Structured reaction media such as regular or inverse micelles (Qi et al 1997; Tanori and Pileni 1997; Li et al 1999; Chen et al 2000) as well as electrochemical methods (Yu et al 1997; Chang et al 1999) have also been used successfully for the synthesis of anisotropic gold nanoparticles. Recently, Pastoriza-Santos and Liz-Marzan (2002) have shown the synthesis of silver nanoprisms in dimethylformamide using a polymeric stabilizer. Silver nanodisks have also been formed by sonicating silver ions and hydrazine dissolved in reverse micelles consisting of $d i$ (2-ethyl-hexyl)sulfosuccinate (AOT), isooctane and water (Maillard et al 2002). Yener et al (2002) have reported a process for making nanosized silver platelets using soft templates such as the octylamine-water bilayer system. A micellar system involving cetyltrimethylammonium bromide (CTAB) was adopted to generate triangular nanoplates (with highly truncated corners) and circular nanodisks of silver through a seed-mediated growth process (Chen and Carroll 2002). Maillard and co-workers have recently demonstrated the synthesis of silver nanodisks by varying the ratio of surfactant AOT to the reducing agent (hydrazine) in reverse micelle solution made of $\mathrm{Ag}(\mathrm{AOT})$ and $\mathrm{Na}(\mathrm{AOT})$ in water and isooctane mixtures (Maillard et al 2002, 2003). Shape transformations have 
also been the focus of recent studies. Sun et al (2003) have reported the transformation of silver nanospheres into nanobelts and triangular nanoplates through a thermal process. Nanorods of silver (Chung et al 1998; Jana et al 2001), gold (Esumi et al 1995; Brown et al 2000; Johnson et al 2002), CdSe (Peng et al 2000), tungsten sulfide (Nikitenko et al 2002), and nanoprisms of silver (Jin et al 2001), gold (Malikova et al 2002), and CdS (Pinna et al 2001) are some of the other interesting nanocrystalline shapes that may now be routinely synthesized in the laboratory. Insofar as metal nanowires/rods are concerned, experimental protocols based on templating (van der Zande et al 1997; Nicewarner-Pena et al 2001), photochemistry (Esumi et al 1995), seeding (Murphy and Jana 2002; Sun and Xia 2002) and electrochemistry (Yu et al 1997; Chang et al 1999) have been developed.

An exciting and hitherto considerably underexploited dynamic biomimetic template for crystal growth is foam lamellae (Chen et al 1998). Chen et al (1998) showed that stabilizing surfactants at the air-bubble/solution interface in foams could be used as nucleation centres for the growth of glycine and $\mathrm{CaCO}_{3}$ crystals. Liquid foams essentially consist of very high density of gas bubbles dispersed in a liquid. The bubbles are stabilized in the liquid matrix by surfactants that adsorb at the gas/liquid interface and are separated by a thin layer of water that, depending on the drainage of the foam etc can approach nanoscale thicknesses. We recognize that the structure of the foam thus consists of bubbles and bilayers of surfactants that are rather similar to two Langmuir monolayers separated by a water channel (scheme 1). By using ionizable surfactants in the foam, it should be possible to electrostatically attach metal ions to the surfactants and thereafter, carry out interesting chemistry to yield a large number of materials over a range of chemical compositions formed directly in the foam.

In this article we describe a novel, simple procedure for the synthesis of anisotropic silver nanoparticles in aqueous foams stabilized by the anionic surfactants (AOT) and sodium dodecyl sulphate (SDS). This is accomplished in a two-step process involving the electrostatic binding of $\mathrm{Ag}^{+}$ions with AOT/SDS molecules at the air-bubble solution interface in the foam followed by reaction with $\mathrm{NaBH}_{4}$ solution in the final step. The silver ions are reduced in the foam that acts as a template and results in the formation of silver nanoparticles of different morphologies that we believe originate in structurally different regions of the foam (scheme 1). Our interest in developing a foam-based method for the synthesis of nanomaterials stems from the fact that the large internal surface area of the foam can be utilized to synthesize large amounts of nanomaterials in a single step and thus
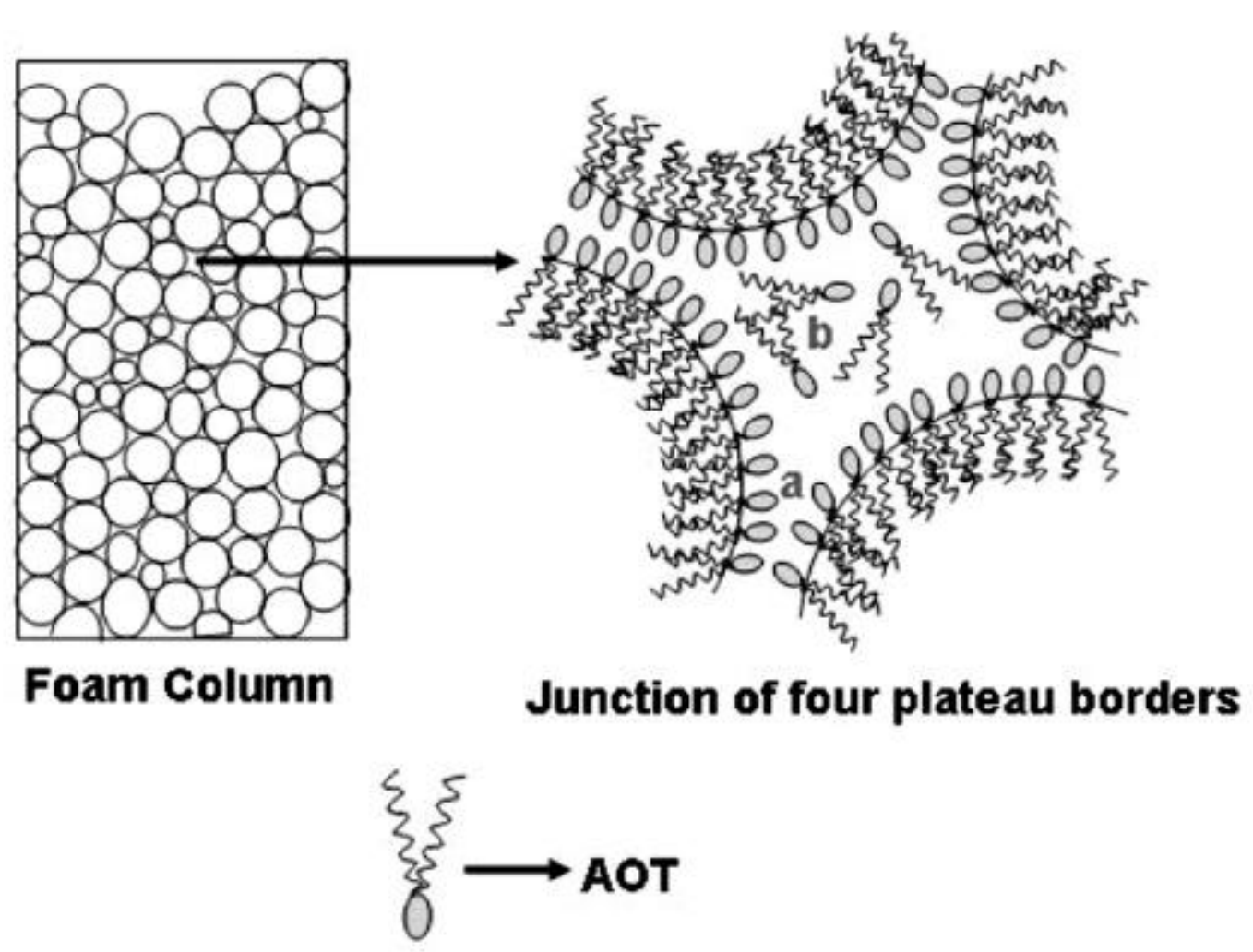

Scheme 1. Cartoon showing different regions in the foam believed to be involved in the synthesis of silver nanocrystals of variable shape. The regions ' $a$ ' and ' $b$ ' are described in the text. 
provide a suitable method for scale-up. For example, there is a growing demand for large-scale production of $\mathrm{CaCO}_{3}$ nanoparticles for application in polymer, paper and cosmetics industries where such foam-based methods could be important.

\section{Experimental}

\subsection{Chemicals}

Silver sulphate $\left(\mathrm{Ag}_{2} \mathrm{SO}_{4}\right)$, aerosol OT (sodium bis-2-ethylhexyl-sulfosuccinate, AOT), sodium dodecyl sulphate (SDS) and sodium borohydride $\left(\mathrm{NaBH}_{4}\right)$ were obtained from Aldrich Chemicals and used as-received.

\subsection{Preparation of silver nanocrystals in aqueous AOT/SDS foam}

In a typical experiment, a rectangular column of $50 \mathrm{~cm}$ height and a square base of $10 \times 10 \mathrm{~cm}^{2}$ with sintered ceramic discs embedded in it were used for generation of the foam. An aqueous mixture of $100 \mathrm{ml}$ of $5 \times 10^{-3} \mathrm{M}$ silver sulphate solution and $100 \mathrm{ml}$ of $5 \times 10^{-3} \mathrm{M}$ AOT was taken in the rectangular column and the foam built up by injecting air at a pressure of 1-5 psi through a porous ceramic disc fixed to the bottom of the foam column. Stable foams of $40 \mathrm{~cm}$ height and with bubble size of $1 \mathrm{~mm}$ could routinely be obtained. After carefully draining out the excess aqueous $\mathrm{AOT}+\mathrm{Ag}_{2} \mathrm{SO}_{4}$ solution, the silver ions in the foam were subjected to reduction by capillary flow of sodium borohydride solution into the foam. As the silver ions were reduced and nanoparticle formation progressed, the foam changed to a yellowishbrown colour and gradually collapsed. The collapsed foam solution containing silver nanoparticles was collected through an outlet provided at the bottom of the column (sample 1). This solution (sample 1) was then subjected to centrifugation at $5000 \mathrm{rpm}$ for $30 \mathrm{~min}$ following which the pellet (sample 2) and supernatant (sample 3) were separated and characterized by different techniques. Please note that sample 2 for further analysis was prepared by redispersing the silver nanoparticle pellet in $10 \mathrm{ml}$ of water. To understand the effect of foam structures on the morphological differences of silver nanoparticles, we have repeated the experiment using SDS instead of AOT as the foam-stabilizing surfactant. The experiments with the SDS foam are identical to that for AOT. The silver nanoparticle foam solution in this case is designated as sample 4.

\section{$2.3 U V$-vis spectroscopic studies}

The optical properties of AOT and SDS-capped silver nanoparticle solutions (samples 1-4) were monitored on a Hewlett-Packard diode array spectrophotometer (model HP-8452) operated at a resolution of $2 \mathrm{~nm}$.

\section{$2.4 \quad X$-ray diffraction measurements}

X-ray diffraction (XRD) analysis of drop-coated films on glass substrates from the AOT-capped silver nanoparticles in sample 1 was carried out on a Phillips PW1830 instrument operating at $40 \mathrm{kV}$ and a current of $30 \mathrm{~mA}$ with $\mathrm{CuK}_{\alpha}$ radiation.

\subsection{Fourier transform infrared (FTIR) spectroscopy measurements}

FTIR spectra were recorded from drop-coated films of silver nanoparticles in sample 1 deposited on a Si (111) substrate on a Perkin Elmer Spectrum-One Spectrometer operated in the diffuse reflectance mode at a resolution of $4 \mathrm{~cm}^{-1}$. The spectrum of pure AOT was also recorded for comparison.

\subsection{Transmission electron microscopy (TEM) measurements}

TEM measurements were performed on a JEOL model 1200 EX instrument operated at an accelerating voltage of $120 \mathrm{kV}$. Samples for TEM studies were prepared by placing drops of the silver nanoparticle solutions in samples $1-4$ on carbon-coated TEM grids. The films on the TEM grids were allowed to dry in air for 2 min following which the extra solution was removed using a blotting paper.

\section{Results and discussion}

Figure 1A shows the UV-vis spectra of the AOT-capped silver nanoparticle solutions in samples 1-3 (corresponding to curves 1, 2 and 3, respectively) and from the SDScapped silver nanoparticle solution in sample 4 (curve 4, dotted line). The inset of this figure shows pictures of test tubes (labeled 1-4) that correspond to the silver nanoparticle solutions in samples $1-4$, respectively. The yellow to yellowish-brown colours observed in different solutions are symptomatic of the presence of silver nanoparticles in the solutions. Strong absorption bands are observed to occur at $\sim 400 \mathrm{~nm}$ for all the samples (curves $1-4$, figure 1A). This characteristic resonance corresponds to excitation of surface plasmon vibrations in the silver nanoparticles and is responsible for the striking colours of the different samples (inset, figure 1A). In addition to the sharp absorption band at $400 \mathrm{~nm}$, samples 1 and 2 (curves 1 and 2, respectively, figure $1 \mathrm{~A}$ ) also show the presence of an additional absorption band at higher wavelengths $(\sim 540 \mathrm{~nm})$. There are two possible explanations for this 
long-wavelength band. One is that the AOT-stabilized silver nanoparticles are in a highly aggregated state leading to coupling of the plasmon vibrations between neighbouring particles (Taleb et al 1998; Tan et al 2002). The other possibility is that the silver nanoparticles are anisotropic in shape (Jin et al 2003). In contrast, the silver nanoparticles produced in the SDS foam (curve 4) does not show any obvious broadening of the plasmon vibration band or an additional band at higher wavelengths. The different silver nanoparticle solutions were extremely stable in time with no evidence of aggregation of the particles even after weeks of storage. This indicates significant stabilization of the particles, presumably through surface capping of the silver nanoparticles by the surfactants, AOT/SDS. The differences in the optical properties of the nanoparticle solutions $1-4$, particularly between samples 2 and 3, clearly indicate significant variation in either the state of aggregation of the silver nanoparticles or the shape of the nanoparticles. This issue will be addressed during analysis of TEM micrographs. Figure 1B shows the XRD pattern recorded from a dropcast film of the silver nanoparticles in sample 1. A number of strong Bragg reflections can be seen which correspond to the (111), (200), (220), (311) reflections of fcc silver. The XRD results thus show that the silver nanoparticles formed within the foam are crystalline. Similar diffraction patterns were recorded from samples $2-4$ and have not been shown for brevity.
Figure 2 shows the FTIR spectra in the region 1000$3100 \mathrm{~cm}^{-1}$ recorded from solution-cast films of pure AOT (curve 1) and AOT-capped silver nanocrystals obtained in sample 1 (curve 2). The methylene symmetric and antisymmetric stretching vibrations in both samples are observed to occur close to 2850 and $2920 \mathrm{~cm}^{-1}$, respectively. Absorption bands are also seen at 1054 and $1736 \mathrm{~cm}^{-1}$ in the case of pure AOT (curve 1). The band at $1054 \mathrm{~cm}^{-1}$ is assigned to the $\mathrm{S}=\mathrm{O}$ stretching vibration of the sulfonate group present in the AOT molecules and the band at $1736 \mathrm{~cm}^{-1}$ is due to carbonyl stretch vibrations in the AOT molecules. These bands are shifted to $1062 \mathrm{~cm}^{-1}$ and $1749 \mathrm{~cm}^{-1}$ in the AOT-capped silver nanoparticle film (curve 2) and indicate that surfactant is bound to the silver nanoparticle surface through the hydrophilic sulfonate/carboxylic acid groups. It is also possible that AOT (and indeed SDS) caps the silver nanoparticles via an interdigitated bilayer. At this stage, we are unable to unequivocally make a statement on whether the surfactant exists as a monolayer or bilayer on the surface of the silver nanoparticles.

Figures $3 \mathrm{~A}$ and $\mathrm{B}$ show representative TEM micrographs recorded at different magnifications from a dropcast film of AOT-capped silver nanoparticles in sample 1. Highly anisotropic silver particles with an overall tapelike morphology are seen that co-exist with a small percentage of nearly spherical particles. The higher magnification image (figure 3B) shows the morphology of one
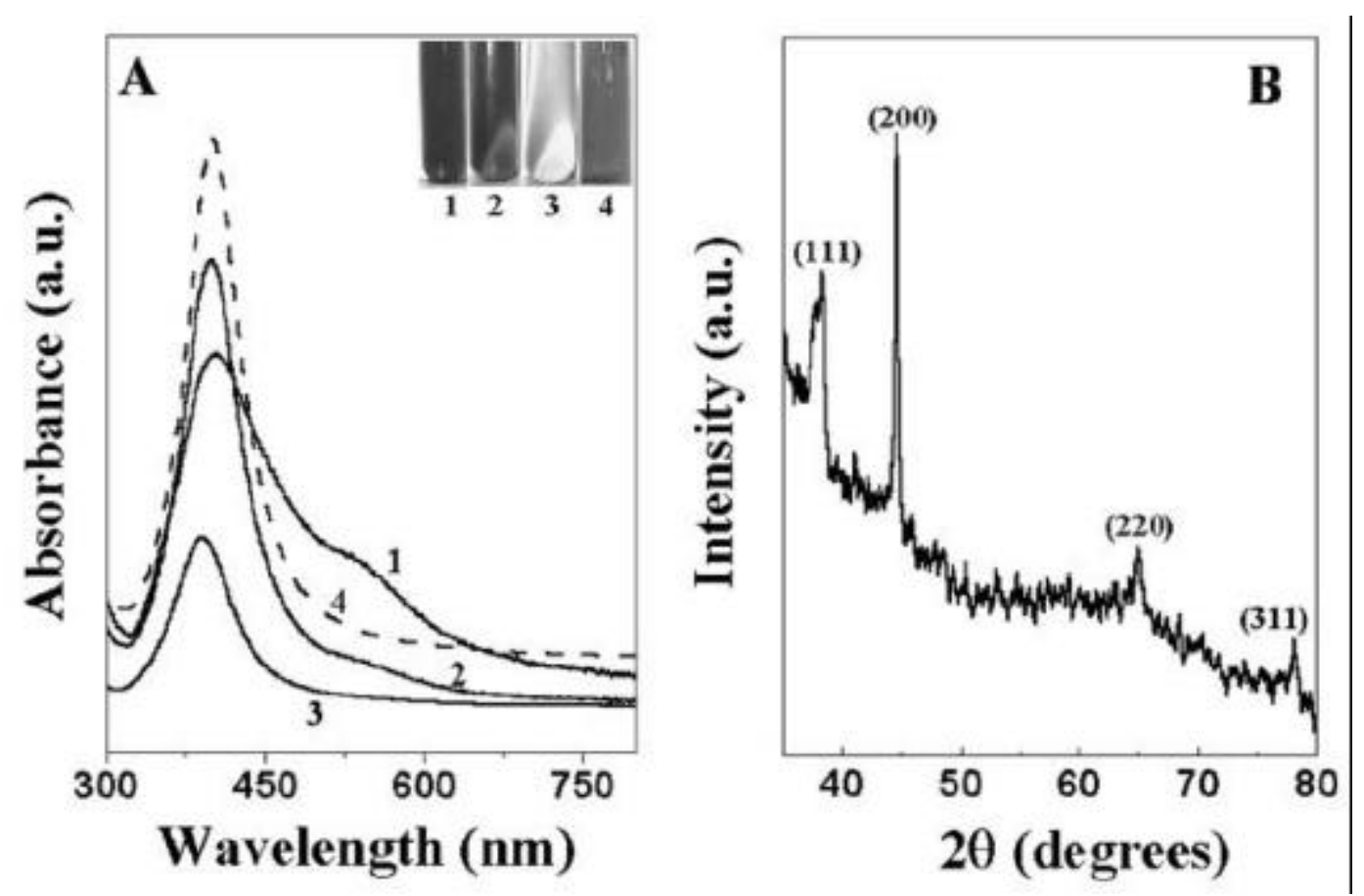

Figure 1. A. UV-vis spectra recorded from sample 1 (curve 1), sample 2 after redispersion in water (curve 2), sample 3 (curve 3) and sample 4 (curve 4, dotted line). The inset shows pictures of test tubes of silver nanoparticle solutions in samples 1-4 and B. XRD pattern recorded from a drop-coated film of sample 1 on glass substrate. 
tape-like silver nanoparticle aggregate in greater detail. The width of the tapes is non-uniform and varies from 5$20 \mathrm{~nm}$. From the TEM measurements, it is clear that the additional absorption band at $540 \mathrm{~nm}$ in the UV-vis spectrum of the AOT-capped silver nanoparticles in sample 1 (figure 1A, curve 1) can be attributed to the flat, anisotropic structure of the nanoparticles and not to aggregation of the particles. The inset of figure 3B shows the selected

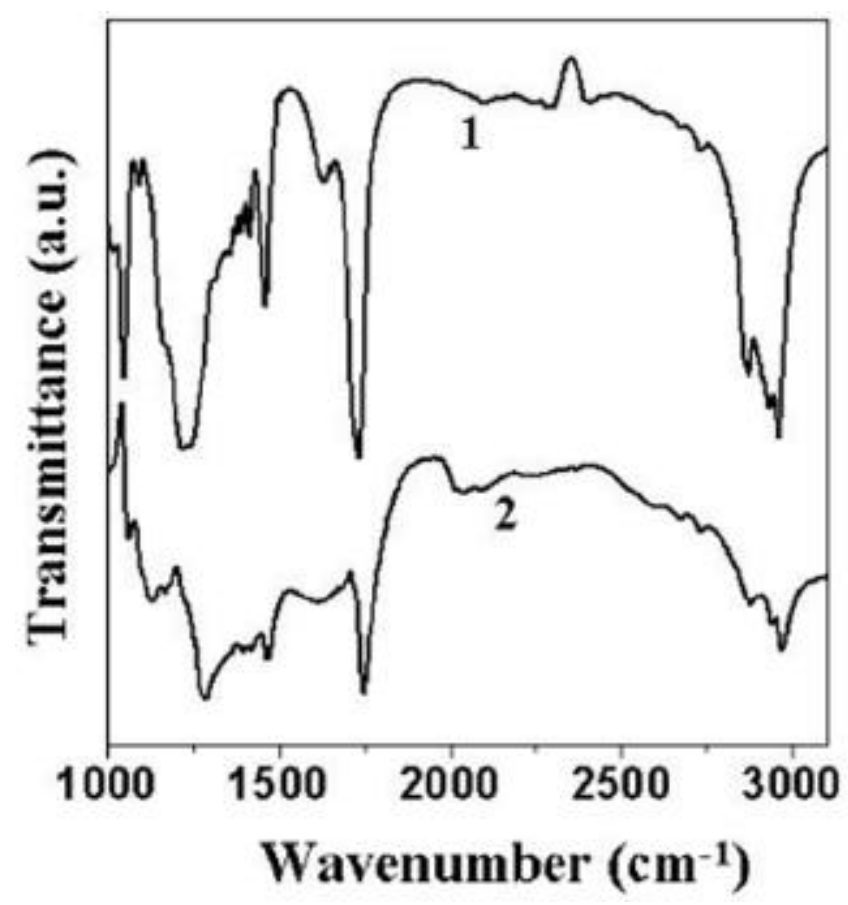

Figure 2. FTIR spectra recorded from a drop-coated film of pure AOT (curve 1) and sample 1 on Si (111) substrates (curve 2). area diffraction (SAED) pattern recorded from the silver nanotapes shown in figure $3 \mathrm{~B}$. The sharp spots in the SAED pattern indicate that the flat silver nanotapes are single crystalline in nature. The diffraction spots marked by circles correspond to the (111) reflections while those marked by squares arise from the (200) reflections from $f c c$ silver.

Figures $4 \mathrm{~A}$ and $\mathrm{B}$ show representative TEM micrographs recorded from a drop-cast film of silver nanoparticles in sample 2. To recollect, this sample is prepared by centrifugation of the as-prepared AOT-capped silver nanoparticles in the foam followed by redispersion of the pellet in water. Highly irregular and extremely flat silver nanosheets/flakes can be seen in these TEM micrographs. That these silver nanosheets are extremely thin is indicated by the fact that buckling of the particles is observed as strain features in the nanosheets. A region where the particles overlap leads to increased contrast and is an additional indication that the silver nanosheets are quite thin. There was no evidence of the presence of spherical silver nanoparticles in this sample indicating that the centrifugation was successful in removing the spherical structures seen in the as-prepared AOT-capped silver nanoparticles (figure 3). The silver nanosheets vary in size from 20-90 $\mathrm{nm}$ and appear to be made of triangular silver nanostructures. The inset of figure $4 \mathrm{~B}$ shows the SAED pattern recorded from the silver nanosheets shown in figure 4B. It is clear from the SAED pattern that the silver nanosheets are single crystalline and have been indexed on the basis of $f c c$ structure of silver. The diffraction spots marked by circles correspond to the (111) reflections while those circumscribed by squares are due to (200) reflections. While there are a large number of reports on the synthesis of silver nanodisks and triangles
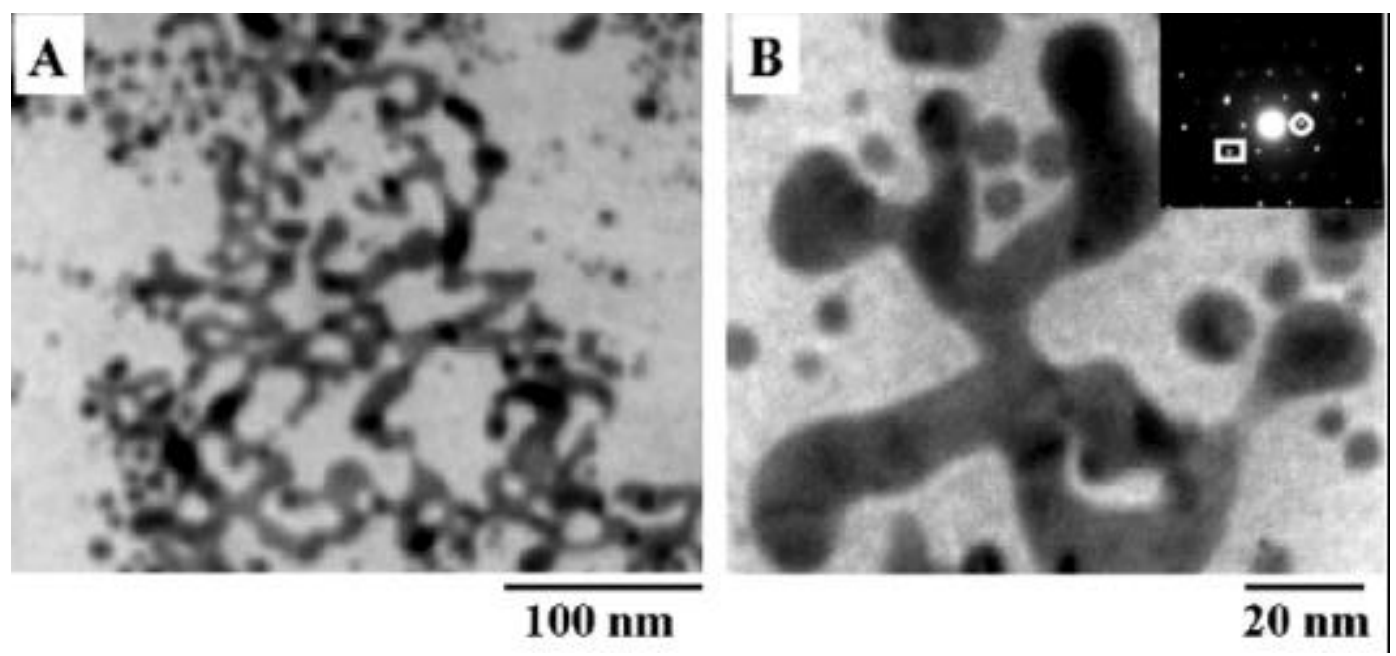

Figure 3. A and B. Representative TEM micrographs recorded at different magnifications from dropcast films of silver nanoparticles from sample 1. The inset of $\mathbf{B}$ shows the selected area electron diffraction (SAED) pattern recorded from the flat-silver nanotapes in the figure. 
(introductory section), to the best of our knowledge there are no reports on the synthesis of single crystalline silver nanosheets in the manner observed in our present study.

Figures $5 \mathrm{~A}$ and $\mathrm{B}$ show representative TEM micrographs recorded at different magnifications from a dropcast film of AOT-capped silver nanoparticles in sample 3 (the supernatant obtained by centrifugation of sample 1). A significant percentage of predominantly spherical silver nanoparticles are seen in these TEM micrographs. The spherical particles are reasonably uniform and range in size from 5-40 nm. Thus, centrifugation leads to separation of the relatively lighter spherical silver nanoparticles in the supernatant while the heavier nanotapes/nanosheets accumulate in the pellet (figure 4).

Figures $6 \mathrm{~A}$ and $\mathrm{B}$ show representative TEM micrographs recorded from a drop-cast film of SDS-capped silver nanoparticles in sample 4. Highly anisotropic silver particles with plate-like morphology are seen that coexist with a small percentage of nearly spherical particles. In gross detail, the nature of silver nanoparticles formed in the SDS foam is similar to that of the AOT foam (figures 3-5) suggesting that the templating mechanism leading to the morphology variation is identical in both cases. On a finer scale, the silver nanoparticles in sample 4 appear to be thicker than those obtained in the AOT foam. While both AOT and SDS are anionic, AOT is a twin-tailed surfactant and this may contribute to variation in the fine structure of the foam. These aspects will be studied in greater detail in forthcoming reports. The inset of figure 6A shows the SAED pattern recorded from silver nanoparticles as shown in figure 6A. The silver nanostructures are clearly polycrystalline. The diffraction rings in the figure could be indexed on the basis of the $f c c$ structure of silver.
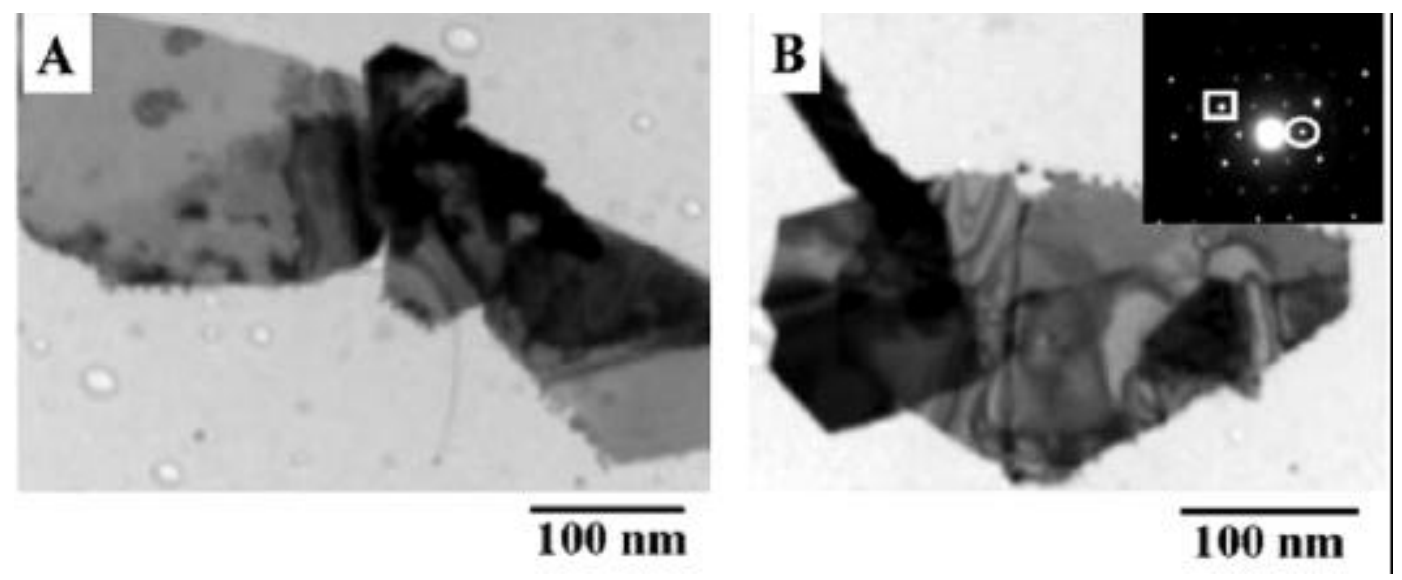

Figure 4. A and B. Representative TEM micrographs from drop-cast films from sample 2 after redispersion in water. The inset of $\mathbf{B}$ shows the selected area electron diffraction (SAED) pattern recorded from the silver nanosheets in this figure.
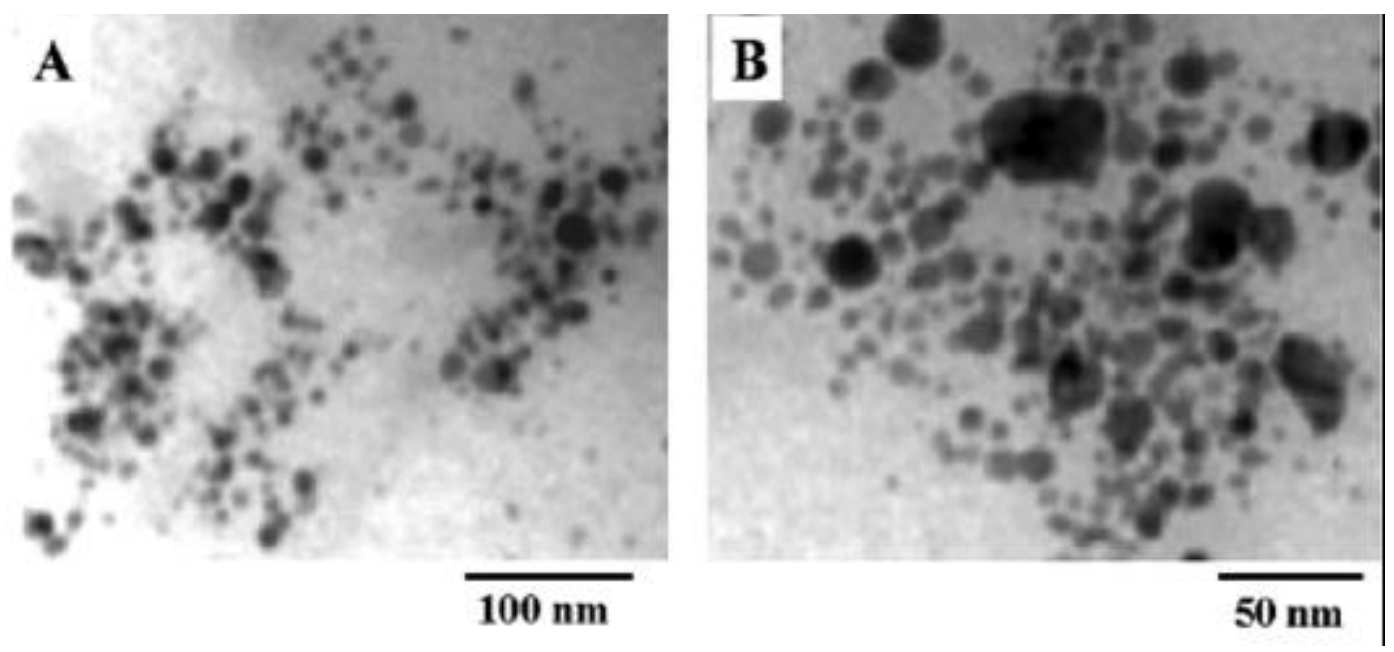

Figure 5. A and B. Representative TEM micrographs recorded at different magnifications from dropcast films of sample 3 . 


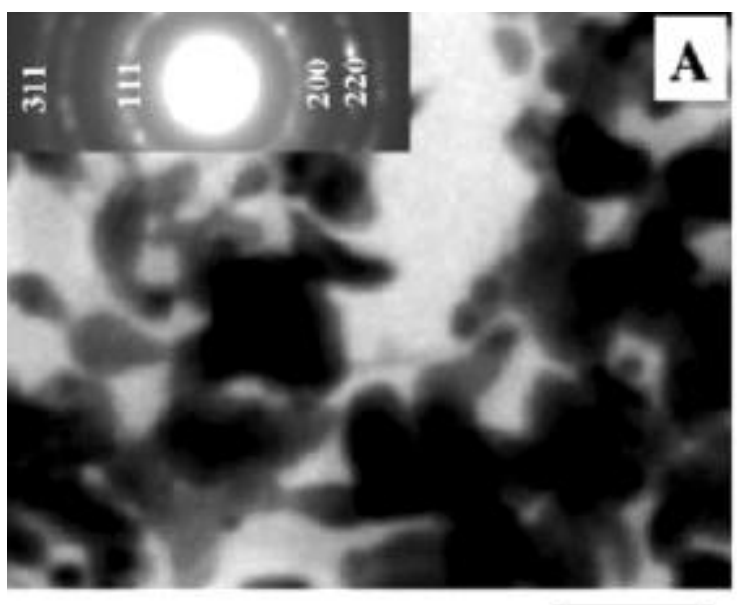

$100 \mathrm{~nm}$

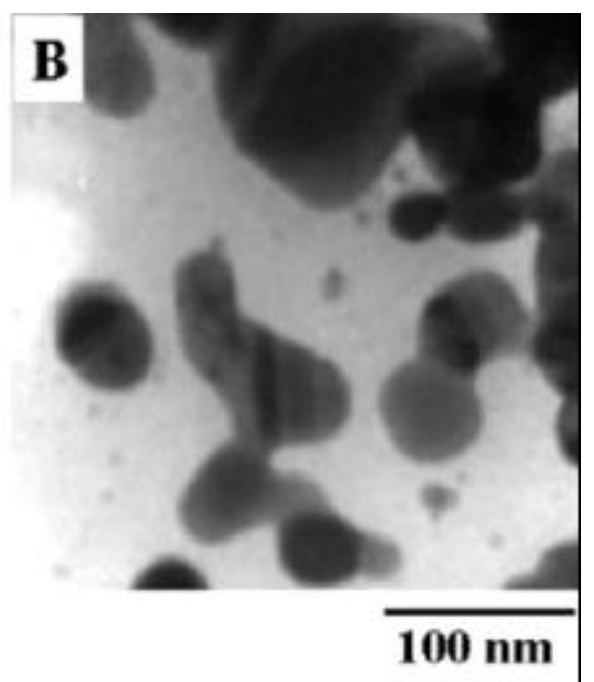

Figure 6. A and B. Representative TEM micrographs recorded from drop-cast films of silver nanoparticles in sample 4. The inset of A shows the selected area electron diffraction (SAED) pattern recorded from the flat-silver nanoplates in this figure.

In scheme 1 we illustrate a possible mechanism operative in the foam that could explain the differences in morphology of the silver nanoparticles grown. The liquid lamellae between bubbles in the foam may be considered to consist of two Langmuir monolayers of AOT electrostatically complexed with $\mathrm{Ag}^{+}$ions and therefore, the silver ions are amenable to borohydride reduction at only the interface. Region ' $a$ ' in scheme 1 shows the liquid lamellae within the plateau borders of the foam wherein the thickness of this region is very small. It is likely that this is the nanoreactor where formation of flat silver nanoplates by borohydride reduction of $\mathrm{Ag}^{+}$ions occurs. Region ' $b$ ' shows the junction of four plateau borders where the reaction space for formation of silver nanoparticles is more extended and isotropic. We believe that the spherical silver nanoparticles form in this region of the foam. However, we caution that much work is required before a full appreciation of the capabilities of the nanoreactors in the foams is achieved. The schematic illustrates the case of silver ion binding in AOT-stabilized foams but in gross detail, is expected to be similar for the foams stabilized with SDS molecules as well.

\section{Conclusions}

The formation of silver nanocrystals in liquid foams stabilized by the surfactants, AOT and SDS, has been described. The process of silver ion entrapment in the foam and their in situ reduction results in the formation of silver nanostructures of variable morphology in the foam. The silver nanoparticle morphology varies from spherical to flat plates/tapes and simple centrifugation enables separation of the different shapes. This morphology variation in the silver nanocrystals is believed to occur due to differences in the structure of the foam, which varies from highly anisotropic (liquid lamellae between bubbles) to extended and isotropic (junction between plateau borders). A combination of extremely large interfacial templating area provided by the liquid lamellae in foams and the dynamic nature of the foam bubbles makes this method potentially exciting for the large-scale synthesis of other inorganic materials (nanomaterials) and minerals and are currently being attempted.

\section{Acknowledgements}

(SM) thanks the University Grants Commission, Government of India, for a research fellowship. This work was partially sponsored by a grant from the Department of Science and Technology (DST), Govt. of India and is gratefully acknowledged.

\section{References}

Alivisatos A P 1996 Science 271933

Berman A D, Ahn J, Lio A, Salmeron M, Reichert A and Charych D 1995 Science $\mathbf{2 6 9} 515$

Brown K R, Walter D G and Natan M J 2000 Chem. Mater. 12 306

Chang S S, Shih C W, Chen C D, Lai W C and Wang C R C 1999 Langmuir 15701

Chen B-D, Cilliers J J, Davey R J, Garside J and Woodburn E T 1998 J. Am. Chem. Soc. 1201625

Chen C C, Chao C Y and Lang Z H 2000 Chem. Mater. 121516

Chen S and Carroll D L 2002 Nano Lett. 21003

Cho A Y 1999 J. Cryst. Growth 2021

Chung S-W, Markovich G and Heath J R 1998 J. Phys. Chem. B102 6685 
El-Sayed M A 2001 Acc. Chem. Res. 34257

Esumi K, Matsuhisa K and Torigoe K 1995 Langmuir 113285 Huang T and Murray R W 2001 J. Phys. Chem. B105 12498

Hu J T, Odom T W and Lieber C M 1999 Acc. Chem. Res. 32435

Jana N R, Gearhart L and Murphy C J 2001 Chem. Commun. 617

Jin R, Cao Y, Mirkin C A, Kelly K L, Schatz G C and Zheng J G 2001 Science 2941901

Jin R, Charles Cao Y, Hao E, Gabriella S M, George C S and Mirkin C A 2003 Nature 425487

Johnson C J, Dujardin E, Davis S A, Murphy C J and Mann S 2002 J. Mater. Chem. 121765

Kamat P V 2002 J. Phys. Chem. B106 7729

Li M, Schnablegger H and Mann S 1999 Nature 402393

Maillard M, Giorgio S and Pileni M-P 2002 Adv. Mater. 141084

Maillard M, Giorgio S and Pileni M P 2003 J. Phys. Chem. B107 2466

Malikova N, Pastoriza-Santos I, Schierhorn M, Kotov N A and Liz-Marzan L M 2002 Langmuir 183694

Murphy C J and Jana N R 2002 Adv. Mater. 1480

Nicewarner-Pena S R et al 2001 Science 294137

Nikitenko S I, Koltypin Y, Mastai Y, Koltypin M and Gedanken A 2002 J. Mater. Chem. 121450
Pastoriza-Santos I and Liz-Marzan L M 2002 Nano Lett. 2 903

Peng X, Manna L, Yang W, Wickham J, Scher E, Kadanavich A and Alivisatos A P 2000 Nature 40459

Pinna N, Weiss K, Urban J and Pileni M-P 2001 Adv. Mater. 13 261

Qi L M, Ma J M, Cheng H M and Zhao Z G 1997 J. Phys. Chem. B101 3460

Sun Y G and Xia Y N 2002 Adv. Mater. 14833

Sun Y, Mayers B and Xia Y 2003 Nano Lett. 3675

Swami A, Kumar A, Selvakannan P R, Mandal S, Pasricha R and Sastry M 2003 Chem. Mater. 1517

Taleb A, Petit C and Pileni M P 1998 J. Phys. Chem. B102 2214

Tanori J and Pileni M P 1997 Langmuir 13639

Tan Y, Jiang L, Li Y and Zhu D 2002 J. Phys. Chem. B106 3131

van der Zande B M I, Bohmer M R, Fokkink L G J and Schonenberger C 1997 J. Phys. Chem. B101 852

Yener D O, Sindel J, Randall C A and Adair J H 2002 Langmuir 188692

Yu Y Y, Chang S, Lee C J and Wang C R C 1997 J. Phys. Chem. B101 6661 